\title{
Effect of Radial Magnetic Field and Temperature Gradient on Separation of Components of a Binary Fluid Mixture Confined in an Annular Region
}

\author{
Bishwaram Sharma \\ Department of Mathematics, \\ Dibrugarh University,Dibrugarh- \\ 786004, Assam, India
}

\author{
Ram Niroj Singh \\ Marwari Hindi High School, \\ Jalukpara, Dibrugarh-786001, \\ Assam, India
}

\author{
Rupam Kr. Gogoi \\ Sibsagar College, Joysagar, \\ Joysagar-785665, \\ Assam, India
}

\begin{abstract}
The effect of a radial magnetic field on separation of a binary mixture of incompressible viscous thermally and electrically conducting fluids confined between two concentric cylinders in which the inner is rotating with a uniform angular velocity and having a uniform suction of the mixture with a constant velocity and outer is at rest. The equations governing the motion, temperature and concentration in cylindrical polar coordinate are solved analytically. The solution obtained in closed form for concentration distribution is plotted against the radial distances from the surface of the inner cylinder for various values of non-dimensional parameters viz. The Hartmann number, thermal diffusion number, baro diffusion number, suction Reynolds number, the product of Pradtl number and Eckert number, the product of Schmidt number and suction Reynolds number and the suction parameters affects the species separation of rarer and lighter component significantly
\end{abstract}

\section{Keywords}

Binary mixture, incompressible fluid, thermal diffusion, baro diffusion, magnetic field

\section{INTRODUCTION}

The presence of temperature gradient separates the two components of a binary fluid mixture which is required in many industrial processes. In the atmosphere also separation of various components of gases takes place due to the existence of temperature gradient. Liu [1] has discussed the flow of a binary mixture of gases of unequal molecular weights over the surface of a heated wall with uniform suction. Hurle and Jakeman [2] has discussed the diffusion effect in a binary mixture on which temperature and concentration gradients are imposed. They have found that the concentration gradient required to produce significant effects in the mixture so small that it may well be the diffusion of the mixture which is generated by the temperature gradient.

In a binary fluid mixture the diffusion of individual species takes place by three mechanisms namely ordinary diffusion, pressure diffusion (or baro-diffusion) and thermal diffusion. The diffusion flux i of lighter and rarer component is given by Landau and Lifshitz[3] as:

$$
\mathbf{i}=-\rho \mathcal{D}\left[\nabla \mathrm{c}_{1}+\mathrm{k}_{\mathrm{p}} \nabla \mathrm{p}+\mathrm{k}_{\mathrm{T}} \nabla \mathrm{T}\right]
$$

The ordinary diffusion contribution to the mass flux is seen to depend in a complicated way on the concentration gradients of the components present in the mixture. The baro-diffusion indicates that there may be a net movement of the components in a mixture if there is a pressure gradient imposed on the system. Thermal diffusion describes the tendency for species to diffuse under the influence of a temperature gradient. It is shown in ref. Groot and Mazur [4] that if separation due to thermal diffusion occurs then it may even render an unstable system to stable one. This effect is also quite small, but devices can be arranged to produce very steep temperature gradients so that separation of mixtures are effected.

Srivastava[5] has discussed the effects of rotation and temperature on the flow of a binary mixture of incompressible viscous fluids filling the space between two co-axial circular cylinders, the inner one rotates with a constant angular velocity and the outer one is at rest. Many investigators[6-22, 25-30] analyzed the effects of baro-diffusion and thermal diffusion on separation of a binary mixture in different geometries.

In this paper we shall discuss the effect of a radial magnetic field on separation of a binary mixture of incompressible viscous thermally and electrically conducting fluids confined between two concentric cylinders in which the inner is rotating with a uniform angular velocity and having a uniform suction of the mixture with a constant velocity and the outer is at rest.

\section{GOVERNING EQUATIONS AND BOUNDARY CONDITIONS}

We consider here the case when one of the components of the binary mixture of incompressible thermally and electrically conducting viscous fluids is present in small quantity, hence the density and viscosity of the mixture is independent of the distribution of the components. The concentration $c_{2}$ of heavier and more abundant component is given by $c_{2}=1-$ $c_{1}$. The flow problem of the binary mixture is identical to that of a single fluid, but the velocity is to be understood as the mass average velocity $\mathbf{V}=\left(\rho_{1} \mathbf{V}_{1}+\rho_{2} \mathbf{V}_{2}\right) / \rho$ and the density $\rho=\rho_{1}+\rho_{2}$, where the subscripts 1 and 2 denote the rarer and the more abundant components respectively. The equation of continuity and the equation of motion of an incompressible fluid in steady case are respectively,

$$
\begin{gathered}
\boldsymbol{\nabla} \cdot \mathbf{V}=0 \\
\text { and } \rho(\mathbf{V} . \boldsymbol{\nabla}) \mathbf{V}=-\boldsymbol{\nabla} \mathbf{p}+\rho \mathbf{F}+\mu \nabla^{2} \mathbf{V}+\mathbf{J} \times \mathbf{B}
\end{gathered}
$$

In steady motion the Maxwell equations are given by

$$
\operatorname{curl} \mathbf{H}=4 \pi \mathbf{J}
$$

$\operatorname{curl} \mathbf{E}=0$

$\operatorname{div} \mathbf{H}=0$ 
It is well known that for most of the fluids used in engineering applications collision frequency exceeds the cyclotron frequency for electrons. As the Hall current factor is ratio of the cyclotron frequency to the collision frequency, so the Hall current is very small and we have neglected in our discussion. Consequently Ohm's law is given by

$$
\mathbf{J}=\sigma[\mathbf{E}+\mathbf{V} \times \mathbf{B}]
$$

where

$$
\mathbf{B}=\mu_{\mathrm{e}} \mathbf{H}
$$

The energy equation in steady case is given by

$$
\mu \mathrm{c}_{\mathrm{p}} \mathbf{V} \cdot \boldsymbol{\nabla T}=\kappa \nabla^{2} \mathrm{~T}+\mu \varphi+\frac{\mathbf{J}^{2}}{\sigma}
$$

where the last term $\frac{\mathbf{J}^{2}}{\sigma}$ represents heat due to electrical resistive dissipation.

The equation of species conservation of the first component is given by (see Landau and Lifshitz [24])

$$
\rho(\mathbf{V} . \nabla) c_{1}=-\nabla . \mathbf{i}
$$

where $\mathbf{i}$ is given by (1). The co-efficients $k_{p}$ and $k_{T}$ may be determined from the thermodynamic properties alone. Landau and Lifshitz [24] have given the explicit expression for the baro diffusion ratio $\mathrm{k}_{\mathrm{p}}$ as

$$
\mathrm{k}_{\mathrm{p}}=\left(\mathrm{m}_{2}-\mathrm{m}_{1}\right)\left[\frac{\mathrm{c}_{1}}{\mathrm{~m}_{1}}+\frac{\mathrm{c}_{2}}{\mathrm{~m}_{2}}\right] \frac{\mathrm{c}_{1} \mathrm{c}_{2}}{\mathrm{p}_{\infty}}
$$

Since $c_{2}=1-c_{1}$ and we have assumed $c_{1}$ to be very small so $c_{1}^{2}$ may be neglected and hence (11) becomes

$$
\mathrm{k}_{\mathrm{p}}=\frac{\left(\mathrm{m}_{2}-\mathrm{m}_{1}\right) \mathrm{c}_{1}}{\mathrm{~m}_{2} \mathrm{p}_{\infty}}=\mathcal{A} \mathrm{c}_{1}
$$

where

$$
\mathcal{A}=\frac{\mathrm{m}_{2}-\mathrm{m}_{1}}{\mathrm{~m}_{2} \mathrm{p}_{\infty}}
$$

The expression $\mathrm{k}_{\mathrm{T}}$ has been suggested Hurl and Jakeman[31]

$$
\mathrm{k}_{\mathrm{T}}=\mathrm{s}_{\mathrm{T}} \mathrm{c}_{1} \mathrm{c}_{2}
$$

For small value of $c_{1}$ (since $c_{2}=1-c_{1}$ and we have assumed $c_{1}$ is very small, so $c_{1}^{2}$ may be neglected) (14) becomes

$$
\mathrm{k}_{\mathrm{T}}=\mathrm{s}_{\mathrm{T}} \mathrm{c}_{1}
$$

Substituting the expression for $\mathbf{i}$ from (1), $\mathrm{k}_{\mathrm{p}}$ from (12) and $\mathrm{k}_{\mathrm{T}}$ from (15) in (10) we get the equation for $c_{1}$ as

$$
(\mathbf{V} \cdot \boldsymbol{\nabla}) \mathrm{c}_{1}=\mathcal{D}\left[\nabla^{2} \mathrm{c}_{1}+\mathcal{A} \boldsymbol{\mathcal { \nabla }} \cdot\left(\mathrm{c}_{1} \nabla \mathrm{p}\right)+\mathrm{s}_{\mathrm{T}} \boldsymbol{\nabla} \cdot\left(\mathrm{c}_{1} \nabla \mathrm{T}\right)\right]
$$

The boundary conditions for velocity $\mathrm{V}=0$ at the outer cylinder and $\mathrm{u}=\mathrm{U}$ at the inner cylinder. The boundary conditions for temperature $\mathrm{T}=\mathrm{T} 0$ at the stationary cylinder and $\mathrm{T}=\mathrm{T} 1$ at the rotating cylinder. The boundary conditions for magnetic field are $b=0$ at both the cylinders. The boundary conditions for the concentration $c_{1}$ are different in different cases. At the surface of a body insoluble in the fluid mixture the total mass flux as well as the individual species flux normal to the surface should vanish [25] i.e.

$$
\rho c_{1} \mathbf{V} \cdot \mathbf{n}+\mathbf{i} \cdot \mathbf{n}=0
$$

where $\mathbf{n}$ is the unit normal drawn at the solid surface directed outwards.

Substituting the expression for i from (1) into in (17), we get

$$
\rho c_{1} V \cdot \mathbf{n}-\rho \mathcal{D}\left[\nabla c_{1} \cdot \mathbf{n}+k_{p} \nabla p \cdot \mathbf{n}+k_{T} \nabla T \cdot n\right]=0
$$

If however, there is diffusion from a body that dissolves in the fluid, equilibrium is rapidly established near its surface and the concentration in the fluid adjoining the plates in this case is the saturation concentration $\mathrm{c}_{0}$ (say); the diffusion out of this layer takes more slowly than the process of solution. The boundary condition at such surface is, therefore

$$
\mathrm{c}=\mathrm{c}_{0}
$$

\section{RMULATION OF THE PROBLEM}

We consider here the steady flow of a binary mixture of thermally and electrically conducting viscous incompressible fluids by using the cylindrical polar co-ordinate system $(r, \theta, z)$. The binary fluid mixture is confined between the walls of two infinite concentric circular cylinders of radii $r=a$ which is rotating with a uniform angular velocity $\Omega$ and having a uniform suction of the mixture with a constant velocity $U$ and $\mathrm{r}=\mathrm{b}$ which is at rest $(\mathrm{b}>\mathrm{a})$. The cylinders are maintained at uniform constant temperature $\mathrm{T} 1$ at $\mathrm{r}=\mathrm{a}$ and $\mathrm{T} 2$ at $\mathrm{r}=\mathrm{b}$. A magnetic field of constant strength B0 is applied in radial direction. As we consider here the flow due to uniform angular velocity $\Omega$ of the inner cylinder and cylinders are of infinite length, so the flow depends only on $\mathrm{r}$. Let $\mathrm{u}, \mathrm{v}, \mathrm{w}$ be the velocity components of the mixture at any point in the directions of $\mathrm{r}, \theta, \mathrm{z}$ respectively. Since the motion is purely rotatory, the axial component of the velocity is zero, i.e. $\mathrm{w}=0$. In this case the equation of motion becomes two dimensional. The velocity component $\mathrm{v}$ in the direction of $\theta$ depends only on the distance from the axis of symmetry i.e. $v=v(r)$.

For the above assumption the governing equations (2), (3), (19) and (16) become

$$
\begin{gathered}
\frac{d u}{d r}+\frac{u}{r}=0, \\
u \frac{d u}{d r}-\frac{v^{2}}{r}=-\frac{1}{\rho} \frac{d p}{d r}+v\left[\frac{d^{2} u}{d r^{2}}+\frac{1}{r} \frac{d u}{d r}\right], \\
u \frac{d v}{d r}+\frac{u v}{r}=v\left[\frac{d^{2} v}{d r^{2}}+\frac{1}{r} \frac{d v}{d r}-\frac{v}{r^{2}}\right]-\frac{\sigma v B_{0}^{2}}{\rho}, \\
\rho g C_{p}\left(u \frac{d T}{d r}\right)=\kappa\left[\frac{1}{r} \frac{d}{d r}\left(r \frac{d T}{d r}\right)\right]+2 \mu\left[\left(\frac{d u}{d r}\right)^{2}+\left(\frac{u}{r}\right)^{2}\right]+ \\
\left(\frac{d v}{d r}-\frac{v}{r}\right)^{2}+\sigma v^{2} B_{0}^{2},
\end{gathered}
$$




$$
u \frac{d C_{1}}{d r}=D\left[\frac{1}{r} \frac{d}{d r}\left(r \frac{d C_{1}}{d r}\right)+S_{T}\left\{\frac{1}{r} \frac{d}{d r}\left(r C_{1} \frac{d T}{d r}\right)\right\}+\right.
$$

$\left.A\left\{\frac{1}{r} \frac{d}{d r}\left(r C_{1} \frac{d p}{d r}\right)\right\}\right]$

and the boundary conditions (18) and (19) become

$$
\begin{aligned}
& u=-U, \quad v=a \Omega, \quad w=0, T=T_{1}, \quad \frac{d C_{1}}{d r}+A C_{1} \frac{d p}{d r}+ \\
& S_{T} C_{1} \frac{d T}{d r}=0 \quad \text { at } r=a, \\
& u=-\frac{a}{b} U, v=0, w=0, T=T_{2}, C_{1}=C_{0} \text { at } r=b
\end{aligned}
$$

By using the assumptions

$$
u=-f a \Omega, v=h a \Omega, p=\rho v \Omega p^{*}, T^{*}=\frac{T-T_{1}}{T_{2}-T_{1}}, C=
$$

$\frac{C_{1}}{C_{0}} \quad$ where $y=\frac{r}{a}$

above equations become:

$$
\begin{gathered}
\frac{d f}{d y}+\frac{f}{y}=0 \\
R_{S}\left(f \frac{d f}{d y}-\frac{h^{2}}{y}\right)=-\frac{d p^{*}}{d y}-\frac{d^{2} f}{d y^{2}}-\frac{1}{y} \frac{d f}{d y}, \\
-R_{S}\left(f \frac{d h}{d y}+\frac{f h}{y}\right)=\frac{d^{2} h}{d y^{2}}+\frac{1}{y} \frac{d h}{d y}-\frac{h}{y^{2}}-M^{2} h, \\
-\tau f \frac{d T^{*}}{d y}=\frac{1}{y} \frac{d}{d y}\left(y \frac{d T^{*}}{d y}\right)+E\left[2\left(\frac{d f}{d y}\right)^{2}+2\left(\frac{f}{y}\right)^{2}+\right. \\
\left.\left(\frac{d h}{d y}-\frac{h}{y}\right)^{2}\right]+E^{2} M^{2} h^{2} \\
\text { and } y \frac{d C}{d y}+t_{d} C y \frac{d T^{*}}{d y}+B_{d} C y \frac{d p^{*}}{d y}+S \alpha C=0
\end{gathered}
$$

where

$$
\begin{gathered}
R_{S}=\frac{a^{2} \Omega}{v}, M^{2}=\frac{\sigma a^{2} B_{0}}{\mu}, \tau=\frac{\rho g C_{p} U a}{\kappa}=P_{r} R_{S}, E= \\
\frac{a^{2} \mu \Omega^{2}}{\kappa\left(T_{2}-T_{1}\right)}=P_{r} E_{c}, B_{d}=A \rho v \Omega, \\
t_{d}=S_{T}\left(T_{2}-T_{1}\right), \alpha=\frac{U}{a \Omega} \text { and } S=\frac{U a}{D}
\end{gathered}
$$

and boundary conditions (25) on velocity, magnetic field, temperature and concentration in terms of dimensionless quantities become

$$
\begin{aligned}
& f=\frac{U}{a \Omega}, h=1, w=0, T^{*}=1, \frac{d C}{d y}+t_{d} C \frac{d T^{*}}{d y}+ \\
& B_{d} C \frac{d p^{*}}{d y}+S f C=0 \quad \text { at } \quad y=1, \\
& f=\frac{U}{b \Omega}, h=0, w=0, T^{*}=0, \\
& C=1 \quad \text { at } \quad y=\frac{b}{a} .
\end{aligned}
$$

\section{SOLUTION OF THE PROBLEM}

The solution of (26) under the boundary conditions (31) is given by $=\frac{\alpha}{y}$. Considering $h=h_{0}+h_{1} M+h_{2} M^{2}$, the solution of (28) under the boundary condition (31) is given by

$$
h_{0}=\frac{A_{0}}{y}+\frac{A_{1}}{y^{R_{S}-1}}
$$

The solution of equation (29), (27) and (30) under the boundary conditions (31) are given by

$$
\begin{gathered}
T^{*}=X_{0} \frac{D_{2}}{y^{\tau \alpha}}+X_{1} \frac{1}{y^{2}}+X_{2} \frac{1}{y^{2 R_{S}-2}}+X_{3} \frac{1}{y^{R_{S}}}+X_{4} \log y+ \\
X_{5} \frac{1}{y^{R_{S}-4}}+X_{6} \frac{1}{y^{R_{S}-2}}+D_{3} \\
p^{*}-p_{y=\frac{b}{a}}^{*}=\left(\frac{\alpha}{2}-\frac{1}{2} \alpha^{2} R_{S}-\frac{1}{2} R_{S} A_{0}^{2}\right)\left(\frac{1}{y^{2}}-\frac{1}{\left(\frac{b}{a}\right)^{2}}\right)- \\
2 A_{0} A_{1}\left\{\frac{1}{y^{R_{S}}}-\frac{1}{\left(\frac{b}{a}\right)^{R_{S}}}\right\}-\frac{A_{1}^{2} R_{S}}{2 R_{s}-2}\left\{\frac{1}{y^{2 R_{S}-2}}-\right. \\
\left.\frac{1}{\left(\frac{b}{a}\right)^{2 R_{S}-2}}\right\}
\end{gathered}
$$

and

$$
C=\left(\frac{y}{\frac{b}{a}}\right)^{-S \alpha} e^{-\left\{t_{d} T^{*}+B_{d}\left(p^{*}-p_{y=\frac{b}{a}}^{*}\right)\right\}}
$$

where $\quad A_{0}=\frac{-\left(\frac{b}{a}\right)^{R_{S}-2}}{1-\left(\frac{a}{b}\right)^{R_{S}-2}}, \quad A_{1}=\frac{1}{1-\left(\frac{a}{b}\right)^{R_{S}-2}}, X_{0}=-\frac{1}{\tau \alpha}$ ,$X_{1}=\frac{2 E}{\tau \alpha-2}\left(\alpha^{2}+A_{0}^{2}\right)$,

$X_{2}=\frac{E A_{1}^{2} R_{S}^{2}}{2\left(R_{e}-2\right)\left(\tau \alpha-2 R_{S}\right)}, X_{3}=\frac{4 A_{0} A_{1} E}{\tau \alpha-R_{S}}, X_{4}=-\frac{E M^{2} A_{0}^{2}}{\tau \alpha}, X_{5}=$ $\frac{E M^{2} A_{1}^{2}}{\left(R_{S}-4\right)\left(\tau \alpha-2 R_{S}+4\right)}, X_{6}=\frac{2 E M^{2} A_{0} A_{1}}{\left(R_{S}-2\right)\left(\tau \alpha-R_{S}+2\right)}$

$D_{2}=\frac{1}{X_{6}\left\{1-\left(\frac{a}{b}\right)^{\tau \alpha}\right\}}\left[1-X_{1}\left\{1-\left(\frac{a}{b}\right)^{2}\right\}-X_{2}\left\{1-\left(\frac{a}{b}\right)^{2 R_{s}-2}\right\}-\right.$

$X_{3}\left\{1-\left(\frac{a}{b}\right)^{R_{s}}\right\}+X_{4} \log \frac{b}{a}-X_{5}\left\{1-\left(\frac{a}{b}\right)^{R_{s}-4}\right\}-$

$\left.X_{6}\left\{1-\left(\frac{a}{b}\right)^{R_{s}-2}\right\}\right]$ and $D_{3}=1-X_{1}-X_{2}-X_{3}-X_{5}-X_{6}-$ $X_{0} D_{2}$

\section{RESULTS AND DISCUSSION}

Equation (32)-(35) are exact solution of equations (26)-(30) subject to boundary conditions (31). If we put $\mathrm{td}=0, \mathrm{Bd}=0$, and $S=0$ in (35) of concentration distribution for the rarer and lighter component of binary fluid mixture, we get $c(y)=1$ for all values of y. From this we can conclude that the separation of species ceases to take place if we neglect the combined effect of temperature gradient, pressure gradient and suction i.e. the magnetic field alone does not affects the process of separation. The flow of the fluid is due to temperature gradient developed in the region between the surfaces of the two cylinders, the pressure gradient produced due to rotation of the cylinders and suction at the inner cylinder. In absence of the temperature gradient, pressure gradient and suction the fluid does not flow and hence equilibrium is established in the whole region. Consequently there is no separation of species in the binary fluid mixture. Our results are found to be in good agreement with the results of researchers ([5], [32], [31], [8][16], [18]-[22], [25], [27]-[29],[33]).

Figure 1 reveals that the concentration of the rarer and lighter component decreases with the increase in the values of $y$. The decrease in the value of $c(y)$ is more prominent in the thin boundary layer near the inner rotating cylinder. Figure 1 also reveals that the concentration of the rarer component 
increases with the increase of the baro-diffusion number $\mathrm{Bd}$. Figure 2 and 3 reveal that the effect of increase in the values of $S$ and $\alpha$ are to increase the concentration of the rarer and lighter component of the binary fluid mixture, which means that more and more lighter component is collected at the surface of the inner cylinder if we increase the suction parameters. It also shows that the concentration ratio of the lighter component at the surface of the inner cylinder increases slowly with the increase of $\mathrm{Bd}$, but increase significantly with the increase of S and $\alpha$.

Figures 4, 5, 6, 7 and 8 reveal that the concentration of the rarer and lighter component decreases by increasing thermal diffusion number, the Hartmann number, product of Prandtl number with the suction Reynolds number, suction Reynolds number and product of Prandtl number with the Eckert number. This suggests that the separation is reduced by increasing the values of the thermal diffusion number, the Hartmann number, Prandtl number, suction Reynolds number and Eckert number.

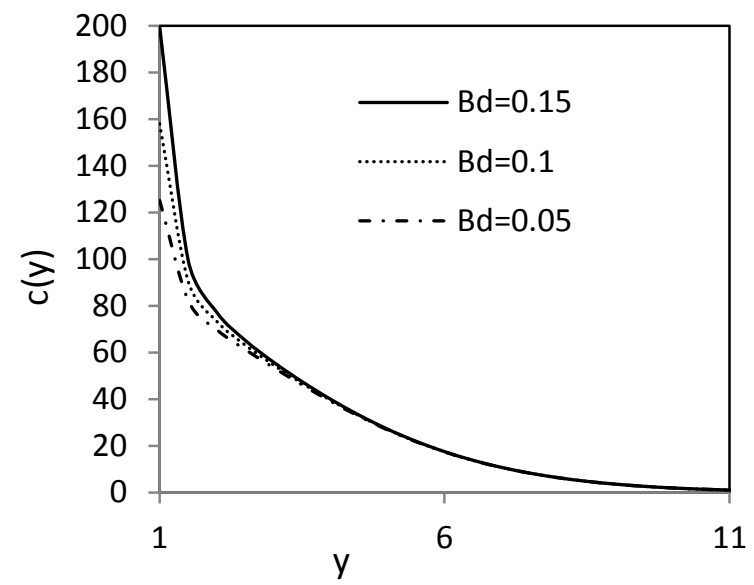

Fig.1. The graph of $\mathrm{c}(\mathrm{y})$ against $\mathrm{y}$ for various values of baro-diffusion number by taking $S=1, \operatorname{Re}=2.5, \alpha=2, \tau=0.01$, $\mathrm{E}=0.5, \mathrm{M}=1$ and $\mathrm{t}_{\mathrm{d}}=\mathbf{0 . 2}$

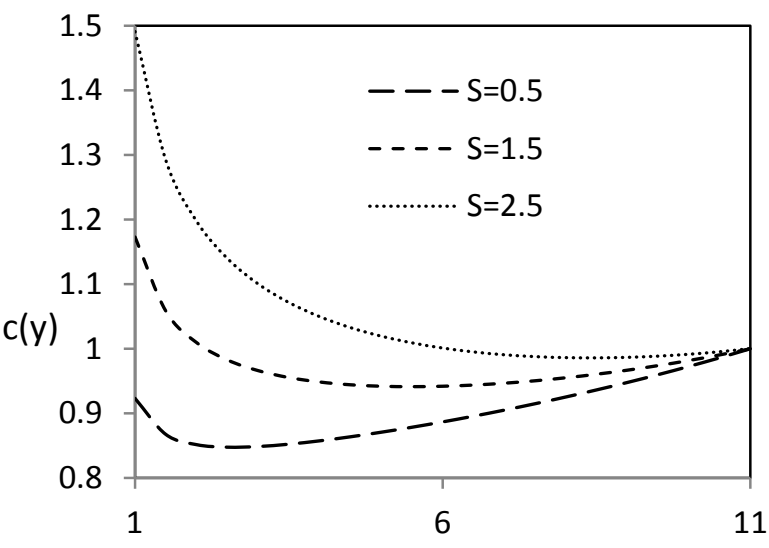

Fig.2. The graph of $\mathrm{c}(\mathrm{y})$ against $\mathrm{y} \% \mathrm{r}$ various values of $\mathrm{S}$ by taking $\operatorname{Re}=0.1, \alpha=0.1, \tau=0.0001, E=.05, M=1, t d=0.02$, Bd $=0.00001$

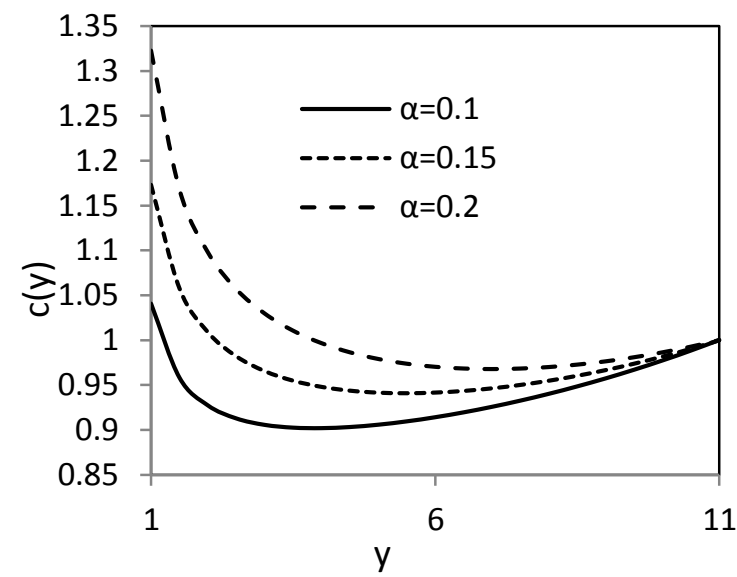

Fig.3. The graph of $\mathrm{c}(\mathrm{y})$ against $\mathrm{y}$ for various values of $\alpha$ by taking $S=1, R e=0.1, E=0.5, \tau=0.0001, M=1, t d=0.2$, Bd=0.00001.

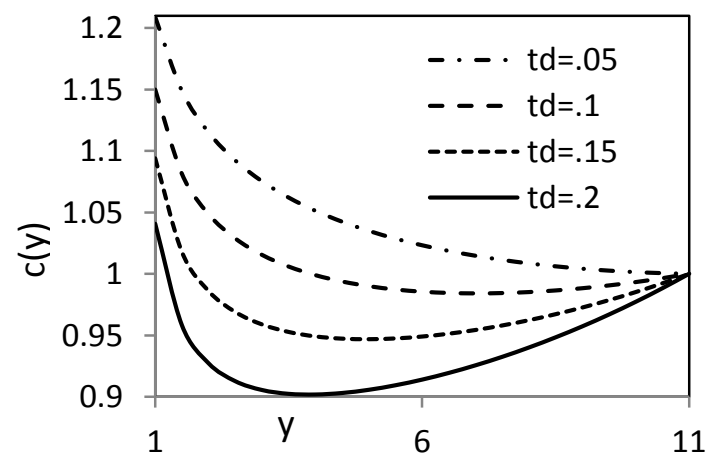

Fig.4. The graph of $\mathrm{c}(\mathrm{y})$ against $\mathrm{y}$ for various values of $\mathrm{td}$ by taking $S=1, R e=0.1, \alpha=0.1, \tau=0.0001, E=0.5, M=1$, Bd $=0.00001$

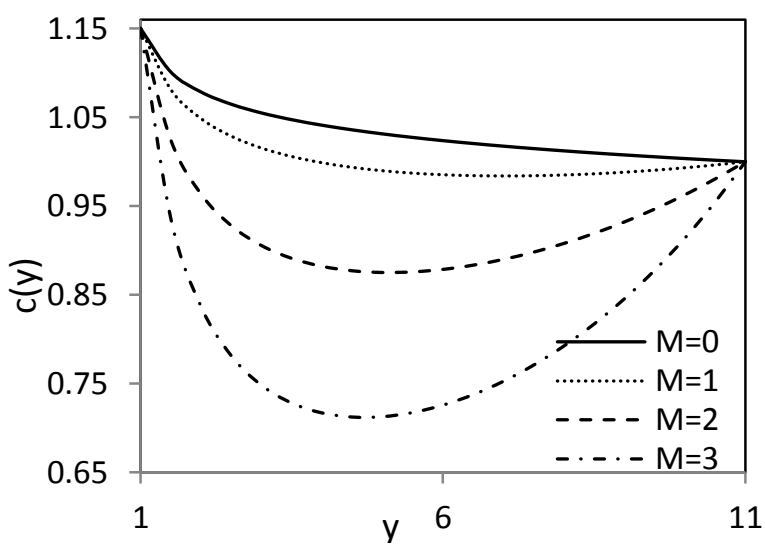

Fig.5. The graph of $\mathbf{c}(\mathbf{y})$ against $\mathbf{y}$ for various values of $M$ by taking $S=1, \operatorname{Re}=0.1, \alpha=0.1, \tau=0.0001, E=0.5, t d=0.1$, Bd=0.00001. 


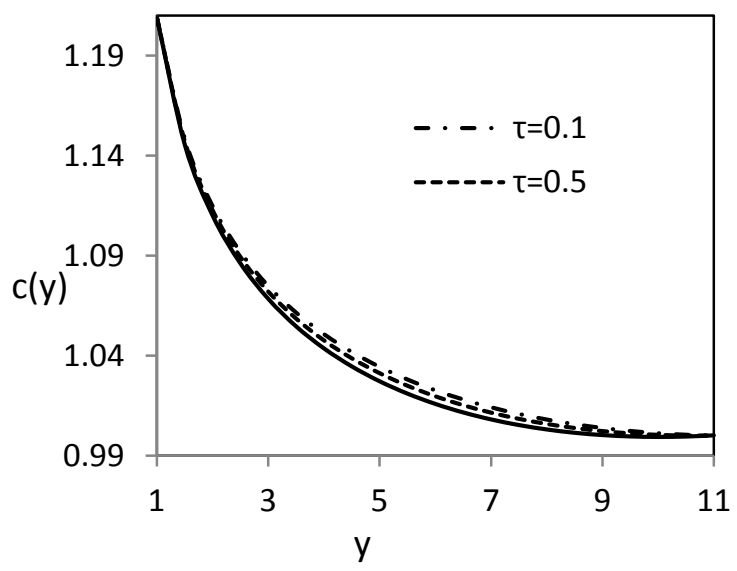

Fig.6. The graph of $\mathrm{c}(\mathrm{y})$ against $\mathrm{y}$ for various values of $\boldsymbol{\tau}$ by taking $S=1, \operatorname{Re}=0.1, \alpha=0.1, E=0.5, M=1, t d=0.05$, Bd=0.00001.

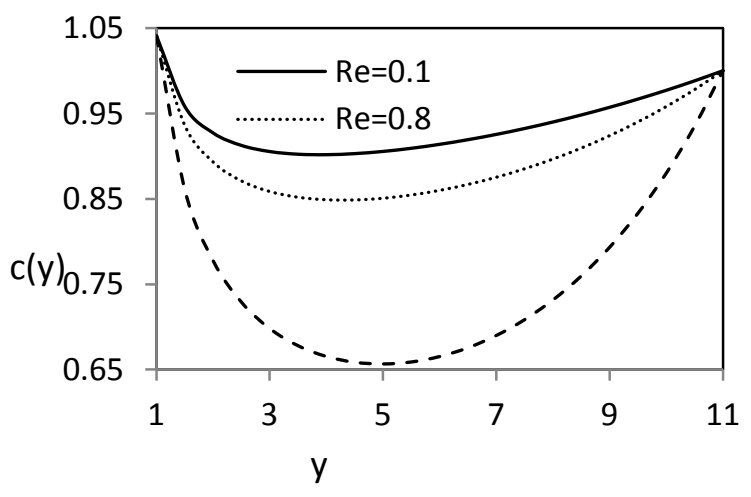

Fig.7. The graph of $\mathrm{c}(\mathrm{y})$ against $\mathrm{y}$ for various values of $\mathrm{Re}$ by taking $S=1, \alpha=0.1, E=0.5, \tau=0.0001, M=1, t d=0.2$, Bd $=0.00001$.

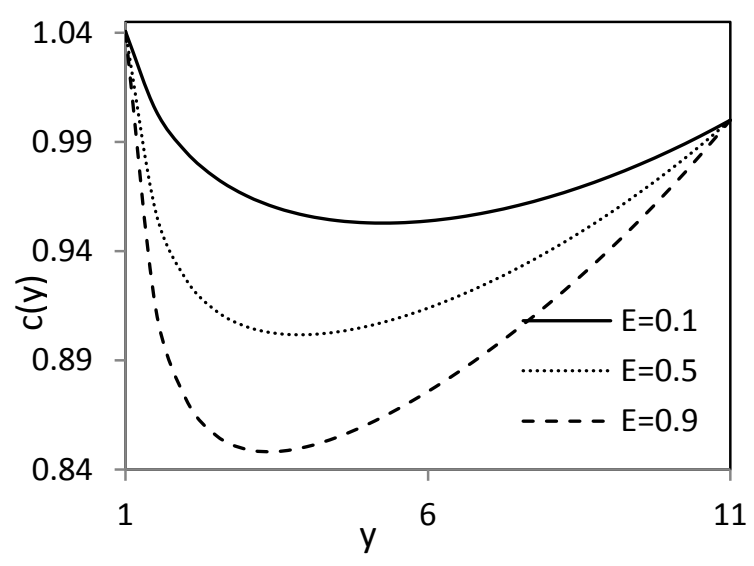

Fig.8. The graph of $\mathbf{c}(\mathbf{y})$ against $y$ for various values of $E$ by taking $S=1, R e=0.1, \alpha=0.1, \tau=0.0001, M=1, t d=0.2$, Bd=0.00001.

\section{REFERENCES}

[1] Liu, V.C. 9 (1959), On the separation of gas mixture by suction of thermo diffusion boundary layer, Quart. J. Mech. And appl. Math. 12, 1-13

[2] Hurle, D.T.J. and Jakeman, E. (1969), Significance of the sorret-effect in the Rayleigh-Jefferey's problem,Phys. Fluids, 12, 2704

[3] Landau LD, Lifshitz EM ( 1960) Electrodynamics of Continuous Media. Pergamon Press, New York

[4] De Groot SR, Mazur P, Mazur S (1962) Non Equilibrium Thermodynamics. North Holland Publishing Co, Amsterdam

[5] Srivastava, A. C. (1992), The effect of rotation and a temperature gradient on the diffusion in a binary mixture, ganita, 43, 163-170.

[6] Poots G (1961) Laminar Natural Convection Flow in Magneto - Hydrodyanamics. Int J Heat Mass Tranfs 3:125

[7] Simon O (1953) an Analysis of Laminar Free Convection Heat Transfer about a Flat Plate Parralle to Direction of the Generating Body Force. N.S.S.A.Rep

[8] Sarma GSR (1975) Barodiffusion in a Roatating Binary Mixture over an infinite Rotating disk. Z Angew Math Phys 26:337- 345

[9] Sharma BR, Hazarika GC(2002) Baro- diffusion and Thermal - diffusion in a Binary Mixture. Nepali Math Sci Report 20(1-2):65-73

[10] Sharma BR (2003) Baro - diffusion and Thermal diffusion in a Binary Mixture of Electrically Conducting Incompressible Fluids due to Rotation of a Circular Cylinder. Bull Pure Appl Sci 22( E1):79-87

[11] Sharma BR(2004) Pressure- diffusion and Soret Effect in a Binary Fluid mixture. Bull Pure Appl Sci 23(E1):165173

[12] Sharma BR, Shah NA (1996) Baro- diffusion and Thermal - diffusion in a Binary Mixture. J Math Phy Sci 30(4):169-179

[13] Sharma BR,Gogoi RK (2000) The Effect Curvature of a Curved Annulus on Seperation of a Binary Mixture. In: Proceeding of the 46th annual technical session, Assam science Society, pp 81-89

[14] Sharma BR, Singh RN (2004) Soret Effect in Generalized MHD Couette Flow of a Binary Mixture. Bull Cal Math Soc 96(5):367-374

[15] Sharma BR, Singh RN (2003) The Effect of Inclination of a Channel on Seperation of a Binary Mixture of Viscous Incompressible Thermally and Electrically conducting fluids. Proc ISTAM 48:36-43

[16] Sharma BR, Shah NA(2002) the effect of pressure gradient and teperature gradient on seperation of a binary mixture of incompressible viscous fluids filling the space outside a uniformly rotating cylinder. In: Proceeding of the 47th annual technical session,vol. 3. Assam Science Society,pp 72-81

[17] Sparrow EM, Cess RD (1961) The effect of a magnetic field on free convection heat transfer. Int J Heat Mass Transf 3:267-274 
[18] Srivastava AC (1985) Barodiffusion in a binary mixture near a stagnation point. Nepali Math Sci Report 10(2):53-62

[19] Srivastava AC(1999)Separation of a binary mixture of viscous fluids by thermal diffusion near a stagnation point. Ganit 50(2):129-134

[20] Srivastava AC (1999) Historical development of fluid dynamicsand mass diffusion in a binary mixture of viscous fluids. Math Stud 68( 1-4):73-85

[21] Srivastava AC ( 1991) seperation of binary mixture in a viscous fluid due to rotating heated sphere. Bull GUMA $6: 63-72$

[22] Srivastava PK(2003) A study of flow of two component fluids, its heat transfer.Ph.D. Thesis, University of Lucknow, Lucknow, India

[23] Globe,S.(1959), Laminar steady state magnetohydrodynamics flow in an annular channel. Phys Fluids 2:404-407

[24] Landau L.D, Lifshitz EM (1959) Fluid Mechanics,2nd edn. Pergamon Press, London

[25] Srivastava AC (1979) Barodiffusion in a binary mixture confined between two disk. Math Forum 2 (2): 16-21

[26] Sharma BR, Hazarika GC, Singh RN (2006) Influence of magnetic field on seperation of a binary mixture in free convection flow considering Soret effect. J natl Acad Math 20:1-20

[27] Sharma BR, Singh RN (2007) Soret effect in natural convection between heated vertical plates in a horizontal magnetic field. J ultra Sci Phy Sci 19 (1)M:97-106

[28] Sharma BR, Singh RN ( 2008) Baro - diffussion and thermal - diffusionin a binary fluid mixture confined between two parallel discs in presence of an small axial magnetic field. Latin Am Appl Res 38:313 - 320

[29] Sharma BR, Singh RN ( 2009) Thermal diffusion in a binary fluid mixture confined between two concentric circular cylinders in presence of radial magnetic field. J Energy Heat Mass Transfer 31:27 - 38

[30] Sharma BR, Singh RN ( 2009) Baro - diffussion and Thermal - diffusionin a Binary Fluid Mixture Confined in an Inclined Channel in Presence of a Weak Magnetic Field. Proc Natl Acad Sci India 79 ( AIII): 273-278

[31] Hurle DT, Jakeman E (1971) Soret - Driven Thermo Solutal Convection. J Fluid Mech 47 (4): 667-687

[32] Sarma GSR ( 1973) Barodiffusion in a Binary Mixture at a Rotating Disk. Z Angew Math Phys 24:789 - 800.

[33] Sharma B R, Singh R N (2010) Separation of species of a binary fluid mixture confined between two concentric rotating circular cylinders in presence of a strong radial magnetic field.Heat Mass Transfer 46:769-777. 